\title{
Left atrial wall imaging using a novel black-blood late gadolinium enhancement sequence
}

\author{
Maxine Tang ${ }^{1,2^{*}}$, Tamer Basha ${ }^{1}$, Sophie Berg ${ }^{1}$, Kraig V Kissinger ${ }^{1}$, Beth Goddu', Warren J Manning ${ }^{1,2}$, Reza Nezafat ${ }^{1}$ \\ From 19th Annual SCMR Scientific Sessions \\ Los Angeles, CA, USA. 27-30 January 2016
}

\begin{abstract}
Background
Late gadolinium enhancement (LGE) is commonly used clinically to identify myocardial infarct or scar as a hyper-enhanced region. However, the blood pool also remains bright in such images, masking adjacent bright tissue. LGE has also been used extensively to image fibrosis in the left atrium (LA) in patients with atrial fibrillation. However, identification of LA wall in LGE is challenging due to the thin wall of LA and high signal of the blood pool in the LA. We recently developed a novel black-blood LGE (BB-LGE) sequence (Fig. 1) which uses an optimized combination of inversionrecovery and $T_{2}$-preparation ( $T_{2}$-prep) prepulses to simultaneously null the healthy myocardium and blood signals by leveraging the higher $\mathrm{T}_{2}$ of blood. In this study, we sought to assess the feasibility of BB-LGE in imaging of LA.
\end{abstract}

\section{Methods}

Seven patients ( 6 male, $65+/-17$ years) with a history of atrial fibrillation were recruited to participate in an IRBapproved study to compare BB-LGE vs. LGE for imaging of the atrial wall. All images were acquired at $1.5 \mathrm{~T}$ (Philips Achieva). LGE and BB-LGE scans were acquired 15-20 minutes after injection of $0.1 \mathrm{mmol} / \mathrm{kg}$ of MultiHance contrast agent. Both sequences were acquired with identical imaging parameters and spatial resolution; $B B-L G E$ required an additional $T_{2}$-prep to null the blood signal. The parameters of the BB-LGE sequence $(\Delta \mathrm{t} 1, \Delta \mathrm{t} 2, \Delta \mathrm{t} 3$ in Fig. 1$)$ were determined in a short $\sim 30 \mathrm{sec}$ navigator scan by a technologist prior to the BB-LGE scan. Images were subjectively scored by an observer according to percentage of the LA border that was clearly defined. A score of 1 was given for scans in which greater than $75 \%$ of the border was well-defined.

${ }^{1}$ Cardiology, Beth Israel Deaconess Medical Center, Boston, MA, USA Full list of author information is available at the end of the article

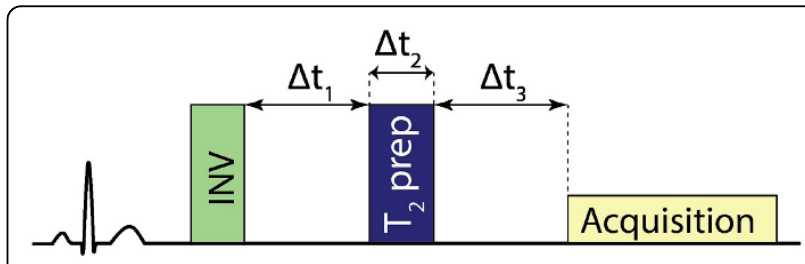

Figure 1 Schematic of the black-blood LGE sequence.

A score of 2 was given for $50-75 \%$, a score of 3 was given for $25-50 \%$, and a score of 4 was given for less than $25 \%$ of the LA border. The scores for each sequence were compared using a paired $\mathrm{T}$-test analysis.

\section{Results}

Figure 2 shows examples of BB-LGE and LGE images of the LA. The LA wall could be easily delineated in BBLGE images. There was significant difference in the scores of LA border definition for the BB-LGE images vs. the LGE images $(p=0.02)$. The BB-LGE image scores were lower than the LGE image scores $(1.38+/$ 0.74 vs. $2.25+/-0.89)$, indicating that a higher percentage of the LA border was well-defined on the BB-LGE images.

\section{Conclusions}

Our proposed BB-LGE sequence has the potential to provide improved visualization of the LA wall compared to LGE protocols due to its ability to simultaneously null both the healthy myocardium and blood pool signals. This preliminary data demonstrates the need for further study of this sequence in patients with suspected LA fibrosis.

\footnotetext{
Authors' details

${ }^{1}$ Cardiology, Beth Israel Deaconess Medical Center, Boston, MA, USA.

${ }^{2}$ Radiology, Beth Israel Deaconess Medical Center, Boston, MA, USA.
} 


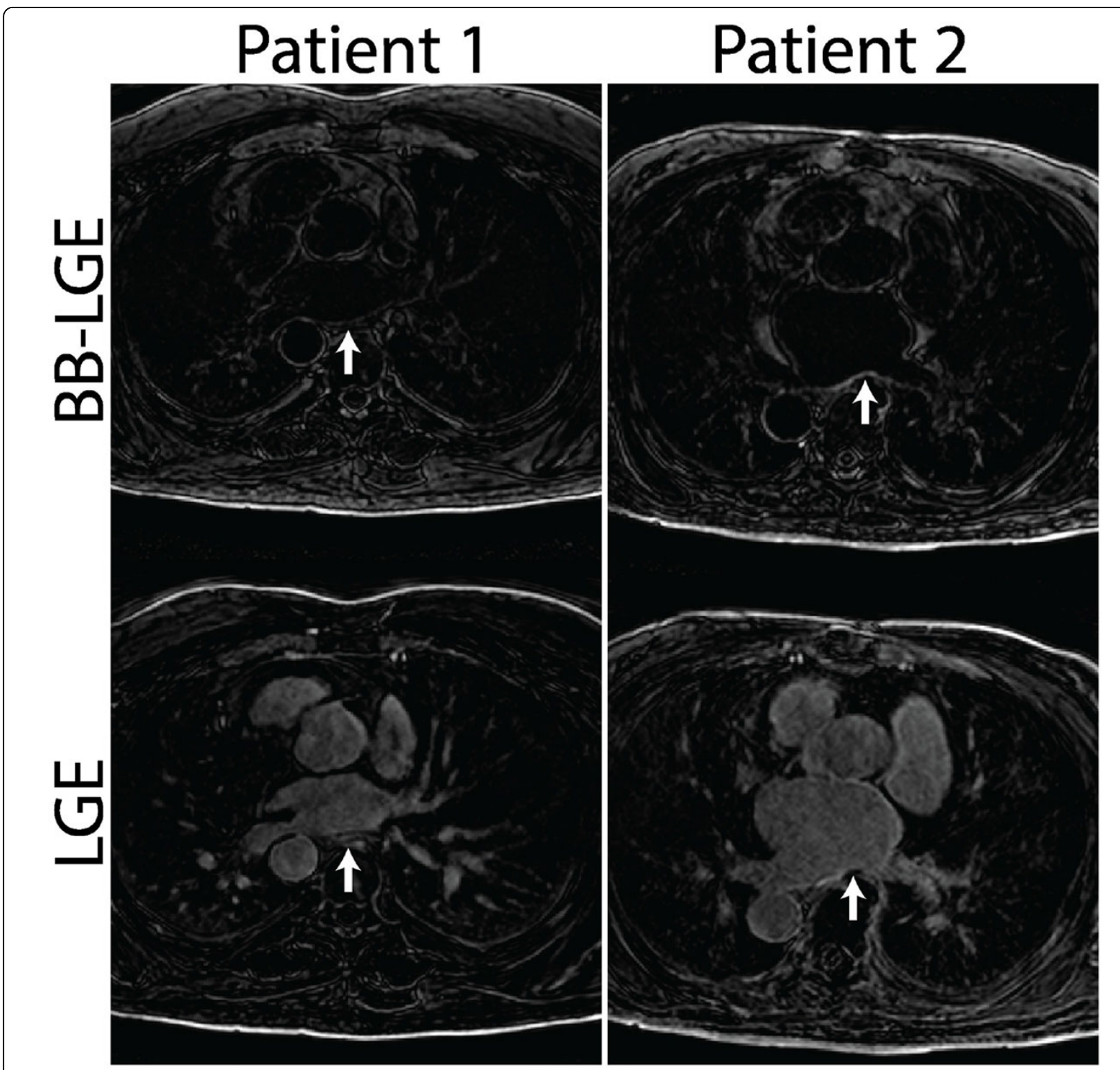

Figure 2 Side-by-side comparison of similar slices showing the left atrium (indicated by white arrow) in three separate patients imaged with both the BB-LGE and LGE sequences.

doi:10.1186/1532-429X-18-S1-P62

Cite this article as: Tang et al:: Left atrial wall imaging using a novel

black-blood late gadolinium enhancement sequence. Journal of

Cardiovascular Magnetic Resonance 2016 18(Suppl 1):P62.
Submit your next manuscript to BioMed Central and take full advantage of:

- Convenient online submission

- Thorough peer review

- No space constraints or color figure charges

- Immediate publication on acceptance

- Inclusion in PubMed, CAS, Scopus and Google Scholar

- Research which is freely available for redistribution 